\title{
ASPA-SKY PATROL FOR THE FUTURE
}

\author{
C. LA DOUS AND P. KROLL \\ Sonneberg Observatory, Sonneberg, Germany
}

\section{Introduction}

Ever since the turn of the century sky patrol observations-i.e., the routine surveillance of the visible night sky - has been one of the main foundations of variable star research, and thus of astronomy in general. Historically several of the major Observatories all around the world were contributing. Up to this day all these observations are in the form of large-field photographic plates, constituting a total of some 2 million plates which, collectively, contain the history of the light changes of celestial objects (mostly in the northern hemisphere and down to some 13 magnitudes apparent brightness or fainter) during the past 100 years. In modern times the last place left in the world where sky patrols are still being carried out routinely is Sonneberg Observatory.

There seems no doubt that the continuation of sky patrol observations is scientifically essential, not only for variable star research, but reaching from observations of near-Earth objects all the way to cosmological ones, and as direct support to space observations. Nevertheless, the current situation is far from satisfactory as the routine production of photographic plates for astronomical usage was discontinued some time ago; Sonneberg, in central Europe, climatically is not an ideal place for astronomical observations; and finally, the extraction of information from photographic plates is difficult and time consuming.

Until not long ago CCD detectors-which have replaced photographic onces in all astronomy for the last two decades-were too small to fully replace the patrol plates. But in the last few years technology has developed sufficiently for sky patrols on the basis of large CCD-arrays to become possible. 


\section{The Aims and Essentials of ASPA}

The idea of ASPA (All Sky Patrol Astrophysics) was born in late 1994 in an attempt to bring sky patrols up to the level of modern technology and to thus fill a badly-needed gap in astronomical observations.

In brief the aims of ASPA are to continue sky patrols using CCD detectors; to construct a world-wide network of fully-automatic telescopes, so-called ASPA-stations; to monitor the entire visible night sky with a time resolution of a few hours; and to make data available on the web within some 24 hours of each observation. In addition, classical photographic observations will be continued until the CCD-based version will be operational. And finally an attempt will be made to scan as many of the old photographic plates as possible and to make them available through the web as well.

For the time being, although the requirements come from astronomy, ASPA is largeley a-rather difficult-technical project, which in turn implies that no observatory on its own is able to put it into practice. Fortunately a collaboration could be started between several departments of the Technical University of Ilmenau, the Optikzentrum in Bochum, and Sonneberg Observatory (all in Germany).

Meanwhile detailed concepts have been prepared for the telescopes (30 $\mathrm{cm}$ diameter, $940 \mathrm{~mm}$ focal length, each equipped with a large CCD array $(7000 \times 9000$ pixels $)$ covering a $5^{\circ} \times 5^{\circ}$ field in the sky, thus providing an angular resolution of around $2^{\prime \prime} /$ pixel; with exposures of some $5 \mathrm{~min}$ duration objects down to $18^{m}-19^{m}$, some $1-2 \times 10^{8}$ objects, and a photometric accuracy of some $3-5 \%$ can be reached), the platform (some 20 such telescopes put together), data transmission to the planned data center in Sonneberg Observatory, data reduction and archiving, and easy access to fully reduced data (light curves or individual points) through the WWW. Funding is being applied for with the Deutsche Forschungsgemeinschaft.

In the fully-grown version ASPA cannot yet be realized with currently available technology - mainly for reasons of the sheer amount of data to be taken care of. For this reason we will start out with just two telescopes and limited info in the web in excess to the very observations, in order to test all the functions and to replace the photographic sky patrol, to begin with in Sonneberg. As technology evolves and money becomes available, little by little the final goal, as described above, will be approached.

More details and regularly updated information are available at http://www.stw.tu-ilmenau.de. 